\title{
Diversity consciousness in the classroom: A case for opening new spaces and generating 'new' knowledge for critical literacy using imaginative practices
}

\author{
Ishwarya N. Iyer ${ }^{1}$ and Sridhar Ramachandran ${ }^{2}$ \\ ${ }^{1}$ School of Education, Indiana University Bloomington, Bloomington, Indiana, USA (ORCID: 0000-0003-3843-3808) \\ ${ }^{2}$ Department of Informatics, Indiana University SE, New Albany, Indiana, USA (ORCID: 0000-0002-3743-3104)
}

\begin{abstract}
The purpose of this research paper is to offer/propose a framework/model of specific imaginative practices, classroom engagement ideas, and implementation pathways that can be adapted across various grade levels within the K-12 (preferably language and literacy) classrooms to utilize the inherent diversity (of lived experiences) amongst the learners as a resources for fostering equity, diversity, and inclusion (EDI) in the classroom while simultaneously generating 'new' knowledge for learning and sense-making. Essentially, the framework proposed will afford expressive outlets into the curriculum via imaginative practices that are designed to allow opportunities for critical literacy learning by bringing common experiences and issues to the fore (utilizing dialogic comprehension-as-sensemaking pedagogy) and thus perpetuating diversity consciousness. The paper also includes a mock-up (i.e. present an example for each step in the framework when possible) of the proposed model in the hope that it will provide an impetus to teacher scholars and educators interested in adapting/including the framework into their classroom.
\end{abstract}

Keywords: Diversity consciousness; Imaginative practices; Critical literacy; Pedagogy

Article History: Submitted 3 January 2019; Revised 2 May 2019; Published online 14 June 2019

\section{Introduction}

In today's globalizing world, the K-12 classroom demographic composition is becoming increasingly diverse. This inherent diversity within the classroom population has the potential to become a pedagogy resource for critical literacy offering "novel ways of thinking and acting" to the learners (and the teachers) along with 'new' opportunities to "broaden their horizons, enter new worlds, become acquainted with a vast range of ideas and perspectives, and reflect on their own perspectives" (Van der Veen, Marjolein, \& Bert, 2017, p. 49). However, classroom diversity as a useful resource for critical literacy inquiry/learning is often unutilized, untapped and/or unrecognized because of the prevalence of the monologic method to classroom instruction (Eranpalo \& Jorgenson, 2018, p. 2). Unfortunately, "a monologically understood world is an objectified world, a world corresponding to a single and unified authorial consciousness" (Hays, 2005, p.9). Sarah Maxine Greene, in her book titled 'Releasing the Imagination', notes (in regards to this authorial consciousness) that "when nothing intervenes to overcome such inertia, it joins with the sense of repetitiveness and uniformity to discourage active learning" $(1995, \mathrm{p} .21)$.

Address of Corresponding Author

Ishwarya N. Iyer, Indiana University Bloomington, School of Education, 201 N. Rose Ave. Bloomington, USA.

$\triangle$ iniyer@iu.edu

How to cite: Iyer, I. N. \& Ramachandran, S. (2019). Diversity consciousness in the classroom: A case for opening new spaces and generating 'new' knowledge for critical literacy using imaginative practices. Journal of Pedagogical Research, 3(2), 63-73. 
Offering a solution, Greene adds that "the classroom situation most provocative of thoughtfulness and critical consciousness is the one in which teachers and learners find themselves conducting a kind of collaborative search, each from her or his lived situation" (1995, p. 23). This call for action (by Greene) to use 'lived situation' for critical literacy and critical consciousness serves as the primary motivation for the framework presented in the paper.

In her book, Greene (1995) also shares her dream/desire for a "humane and liberating classrooms in which every learner is recognized and sustained in her or his struggle" (p. 5). Recognizing that the world is converging, she highlights the need to "socialize diverse young learners" to "counter both relativism and ignorance at once" (p. 9) stating that "one must resist viewing other human beings as mere objects or chess pieces and view them in their integrity and particularity instead. One must see from the point of view of the participant in the midst of what is happening if one is to be privy to the plans people make, the initiatives they take, the uncertainties they face" (p. 10). Echoing similar sentiments, authors Van der Veen, Marjolein, \& Bert (2017 pp. 49-50) in their article titled 'Engaging Children in Dialogic Classroom Talk' state that:

"It requires the effort of teachers to make these classrooms reach their full potential. This is where we (as educators) should decide whether we love the world and our children enough to prepare them to deal with diversity, tensions, and differences; provide them with tools to take advantage of the range of perspectives they encounter; to prepare them to understand the plural other, as well as the plural self (i.e., a multi-voiced self); and to renew a common world that is open and livable".

Taken together, it can be understood that the 'call' is for classroom reform so that dialogic meaning-making and sense-making replaces conventional monologic methods and where classroom diversity (e.g. 'lived situations/experiences') is seen/viewed as a 'curricular' (and not an extra-curricular) resource that can fuel the dialogues within the classroom that in-turn shall promote critical literacy, critical consciousness and social imagination amongst the participants. This ideological shift is necessary (now more than ever) since the goal of education in this globalizing world is to "create citizens of the world: those with the proclivities and abilities to shift across boundaries-geographically, disciplinarily, professionally, and in engagement with others - with a moral and ethical imperative to engage in and sustain equitable and just relations." (Hawkins, 2014, p. 94). In that context, this call for an ideological shift in classroom pedagogy and curriculum implementation opens up two very interesting questions for investigation, viz.:

$>$ Q1. How can the traditional classroom curriculum be transformed into an 'active learning' site where dialogic meaning-making and sense-making helps with opening new spaces for learning and generating new knowledge?

$>$ Q2. Can the diversity in individual 'lived experiences' among the classroom population be utilized as dialogic resource that can promote critical literacy and critical consciousness?

In the remainder of this paper, we will present our exploration/investigation of these two questions in an effort to offer the readers with a sustainable framework that can afford a symbolic 'sample-initiative' towards the envisioned curricular reform (discussed earlier).

\section{Key Concepts, Innovative Ideas and New Directions: Sources of Inspiration}

\subsection{Children as Consumer and Producers of Knowledge}

Medina and Wohlwend (2014) stated that in this era of rapid globalization and transnationalism, the children "live multiliterate lives as they move as consumers and producers of knowledge across real and imagined spaces, across worlds and communities, and in textual diasporas grounded in traveling texts that flow through media, digital spaces, and the consumption structures of global markets" (p. 5). They add that since "reading, writing, and cultural production happen at the intersection of participation in complex worlds and discourses" the children's 'lived experiences' cannot be and should not be "ignored when visualizing literacy pedagogies that 
matter to/for children" (2014, p. 5). They thus make a strong case for utilizing the inherent diversity (that is readily available in the lived experiences of the classroom participants) as a pedagogy (and learning) resource. So, having established that the diversity in lived experiences (diversity) is a valuable resource to learn from, the question then is - how can the lived experience be made part of the classroom discourse?

\subsection{Imaginative Practices: Using Drama Practices for dialogic critical engagements}

Medina, Perry, Lee, and Deliman (2018) draws the attention to the potential and potency with/of using "drama as interpretative meaning making" (p. 2) in the classroom. They add that "the new emerging relationships that are constructed at the intersection of making meaning between texts and drama, become possible in the opportunities drama practices provide to negotiate and explore readers/performers multiple social positionings" (2018, p.3). They state that "(d)rama practices such as hotseating, role-play, tableaux, and human slide shows are some of an always expanding repertoire of performative, embodied, and pedagogical tools that provide opportunities for students to become critical observers and effective problem solvers as they actively and playfully negotiate meaning within and around the text where events take place" (2018, p.5-6). Along the same context, Diamond (1996), in her book titled 'Performance and Cultural Politics', highlights the importance of imaginative practices (like drama and performance) and how it offers the unique opportunity for the participants to don/wear several hats (e.g. writer and performer of personal tales/oral histories, script writer, director etc.). She adds that this opportunity to participate in all phases of a performance (from design to implementation) helps transform the performer's selfconsciousness within politicized spaces. Taken together, these observations by the eminent teacher-scholars presents a strong case for using drama practices as a meaning-making and sensemaking tool to encourage dialogic critical engagements in the classroom towards developing critical consciousness about the diversity around them. Moreover, when the drama is co-written, co-enacted, co-directed and co-produced by the participants in the classroom, it allows for lived experiences to become part of the discourse that then facilitates the opening of "critical spaces within which students negotiate diverse perspectives and generate knowledge that may serve their own educational and social empowerment" (Medina \& Campano, 2006, p. 333). So, having established that drama performances (that utilizes the diversity of lived experiences in its scripts/design/narratives) can facilitate dialogic critical engagements within the classroom, the question then is- how can the teacher initiate reflective discourses to emerge from all learners in the classroom.

\subsection{Self-Excavation as the 'first step': The case for a 'Pre-Text'}

An essential 'first step', before the participants can engage in dialogic exchanges, is for the participants to discover, explore, recognize and articulate their own lived experience (around a topic/issue or everyday experience that the class is exploring) so that they can become self-aware and self-conscious of their unique worldviews before the dialogic engagements begin. This 'first step' is important because it allows every participant to share, document and externalize their otherwise internalized 'position' about an everyday event that the class is planning on exploring thus displaying the diversity upfront. Moreover, this individual self-reflection is essential so that 'original' voices/experiences are allowed to surface free of peer influence and conformity that is common in the socio-political nature of collaborative work. There are many ways this 'first step' can be designed, for example - once the class has decided on an issue/everyday event to investigate/discuss, the teacher can ask each student to first individually create an 'artwork' that identifies with their position on that issue (that is informed by their worldview). The use of art as a medium for contemplative self-reflection will help the student self-excavate internalized issues that can provide insights into how the lived experiences have shaped ones' social imagination. Authors Bhattacharya and Payne, in their paper titled 'Mixing mediums, mixing selves: arts-based contemplative approaches to border crossings', successfully accomplished this 'first step' by using 
imaginative practices like fragmented storytelling, art-making and theorization for self-excavation. In their paper they note the effectiveness of the art-making approach stating:

"Contemplative art-making provides a window, revealing the multiple states of consciousness that arise from experiences, desires, and imagination of possibilities. Through storytelling I am able to attend to what arises in these states of consciousness. I begin to question the aspects of myself I hold as stable and those I separate from myself through resistance. Pushing further, I question how we can attend to what arises when we participate in or resist the effects of social structures of oppression, when the mere act of paying attention automatically places us in opposition, creating a righteous self against an oppressive other" (2016, p. 1114).

The diverse positions that shall emerge from this 'first step' shall become fodder/resource to be used as 'Pre-Text' for the design of the remainder of the imaginative engagements (as discussed in sections 2.1 and 2.2 - also, we will expand on this detail later in this paper). When contemplative art-making is followed up by storytelling, the students will be able to bring to the fore their individual positions about an issue/event that is outside (and uninfluenced) by dominant discourses and that is exactly what is the motto for this 'first step'. This extends the usefulness of the art and also foregrounds meaning making in an integrated and constitutional way into the flow of activities moving away from "linear forms of interpretations" to instead encourage the emergence of meaning making in an "actively negotiated" encounter (Medina et al., 2018, p. 11). Taken together, the sub sections in this section of the paper have successfully explored the feasibility of finding answers to the two research questions presented at the end of the first section. However, before we move on to discussing an implementation framework it is important to discuss (briefly) the role of the teacher, the significance of opening up new spaces and the meaning of diversity consciousness and social imagination as it applies to the context of this paper.

\subsection{Diversity Consciousness as the 'gateway' to Social Imagination}

Diversity Consciousness, as it applies to critical literacy (and especially to the framework to be discussed in this paper), should be interpreted as the critical consciousness about diversity that "helps students (and teachers) develop a flexible, inclusive kind of thinking/awareness of the interconnectedness of our world's different people and cultures" while simultaneously understanding the value "of developing such an awareness" in their "personal life, education, and career" (Bucher, 2000). Development of this critical consciousness is vital because classrooms are getting more "heterogeneous" and so in a classroom devoid of means (and curriculum) to cultivate diversity consciousness "children (and teachers) might experience this melting pot of cultures and perspectives as threatening, confusing, and difficult" (Van der Veen, Marjolein, \& Bert, 2017, p. 49). One way to combat this anxiety (and the associated fear/threat) is to break through the "inertia of habit" and "poke around" investigating the world around paying attention to it and moving "from the habitual and the ordinary and consciously" undertaking a search, instead of "fleeing from it" (Greene, 1995, p. 16 - 24). This search is a precursor to the redevelopment of social imagination "the capacity to invent visions of what should be and what might be" (Greene, 1995, p. 5). In that vein, diversity consciousness is the precursor (and gateway) to initiating/undertaking the search (mentioned above).

\subsection{Teacher as a Co-Participant}

As we seek to integrate imaginative practices into the classroom curriculum to encourage diversity consciousness and re-cultivate social imagination, it is also important to briefly discuss the role of the teacher in this reimagined 'dialogical' space. According to Medina et al. (2018), when imaginative practices as meaning making practices are used in the classroom, the role of teacher has also to be reimagined - "rather than teachers positioning themselves as the facilitator they become co-participants who are an integral part of co-construction of the learning" (p. 16). In that context, authors Van der Veen, Marjolein and Bert (2018) share that in these new dialogic spaces, teachers as co-participants "encourage children to cross the boundaries of their own thinking, of 
their own voiced positions, and try to understand the position of the other. In this process, the different positions or perspectives are negotiated so both the group and the self can progress in thinking" (p. 51). We will conclude this sub section with a quote from Greene's paper (1995) that sums up the importance and significance of the reflective teacher ${ }^{2}$ :

"Teachers imaginative enough to be present to the heterogeneity of social life and to what has been called the "heteroglossia," or the multiple discourses, of the everyday (Bakhtin, 1981) may also have strong impulses to open pathways towards better ways of teaching and better ways of life". (p. 12).

\section{A Framework for fostering Diversity Consciousness in the Classroom}

Now that we have discussed all of the important key concepts that inform and inspire our vision for a dialogic classroom, it is time to share with you our vison in the form of a framework/model of specific imaginative practices, classroom engagement ideas, and implementation pathways that incorporates many (if not all) of the best practices that we have discussed thus far. In this section, we will begin by first presenting our framework visually (See Figure 1) and then discusses each part of the framework (see the labels) is some detail (we will keep it as brief as possible). We will also simultaneously present a mock-up of the framework (i.e. we will present an example for each step in the framework when possible) in the hopes of providing an impetus to teacher scholars and educators interested in adapting/including the framework into their classroom.

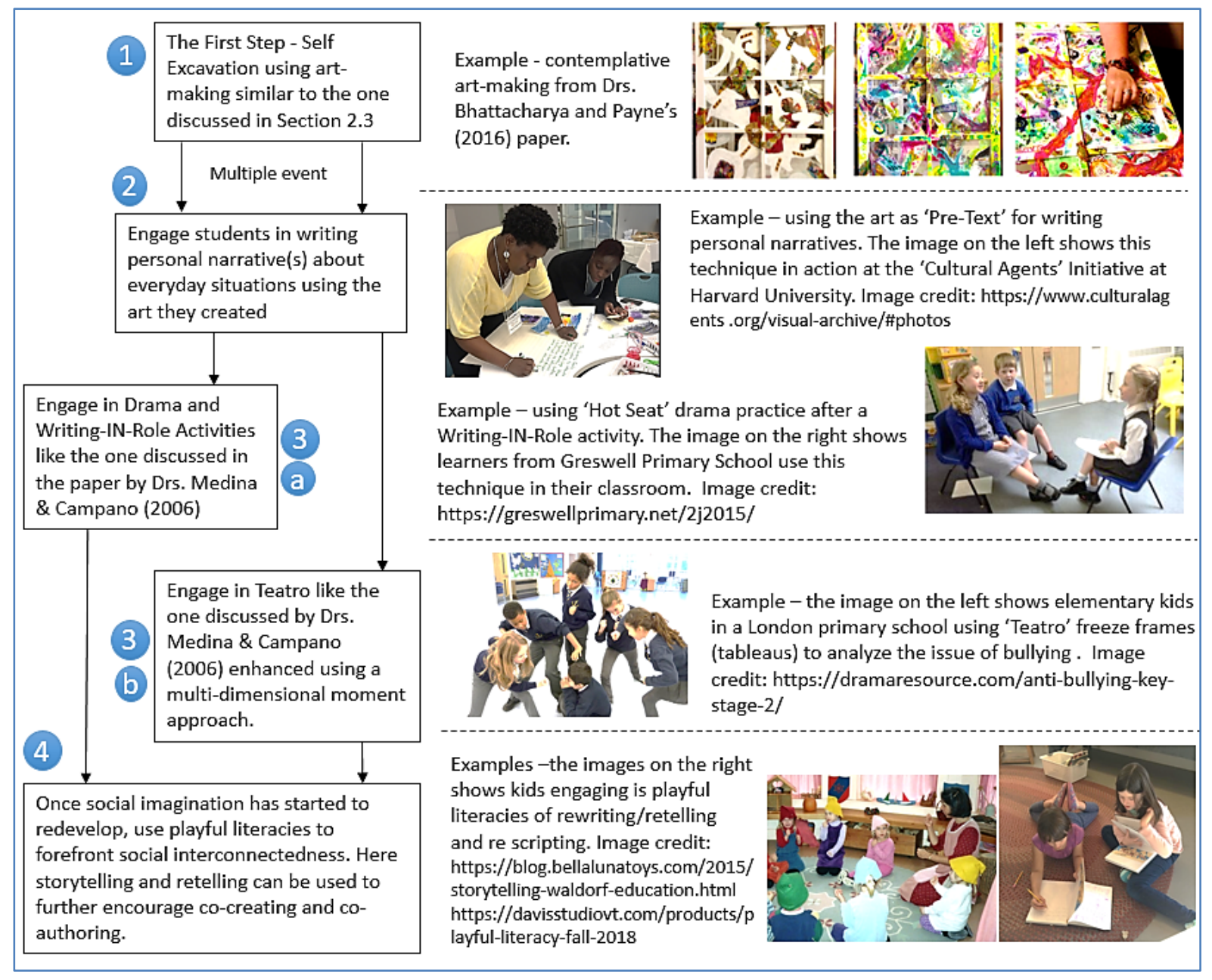

Figure 1. A framework for opening new spaces and generating 'new' knowledge for critical literacy using Imaginative Practices

\footnotetext{
2 In the context of this paper, a reflective teacher is one who has diversity consciousness and the social imagination to properly incorporate imaginative practices into the classroom curriculum.
} 
By design, the framework (shown in Figure1) can be used in any classroom and within any subject area. When using the framework, it activates when the class decides to investigate an issue3/everyday-event/topic together. Thence, the framework should be traversed chronologically (the labels in Figure 1 show the chronological order). After the first pass through the framework with an issue, the framework components can be accessed in any order.

Table 1.

Sample of key drama practices (adapted in modified form from Medina et al. (2018)

\begin{tabular}{ll}
\hline Drama practices & Brief description \\
\hline Freeze frames/tableaus & $\begin{array}{l}\text { Images with no movement representing characters, } \\
\text { tensions and situations in a moment in time. }\end{array}$ \\
\hline Hot seat & $\begin{array}{l}\text { Interviews and questions to one or a group of } \\
\text { characters by the larger community in the drama. }\end{array}$ \\
\hline Writing in role & $\begin{array}{l}\text { Participants write a piece of text as an explicit or } \\
\text { implied characters in the story (a journal, a letter, a } \\
\text { newspaper, a flyer, etc.) }\end{array}$ \\
\hline $\begin{array}{l}\text { Narrations and } \\
\text { dramatizations }\end{array}$ & $\begin{array}{l}\text { Participants narrate or act a story in front of the } \\
\text { audience. }\end{array}$ \\
\hline
\end{tabular}

\subsection{Self-Excavation: Label 1}

As was discussed in section 2.3, this 'first step' in the implementation framework (by design) attends to the call for the need to design expressive outlets into the curriculum via imaginative practices that allow opportunities for bringing individual perspectives (diversity in lived experience) to the fore. This is a very important step because this exercise/step is about respecting the individuality of the participants affording them the opportunity to look inwards at their uniqueness of thought and at how they see/conceptualize the issue/event/topic in the light of their lived experiences. In the context of this first-step, Bhabha notes in his book about "The location of culture' that "the truest eye may now belong to the migrant's double vision" (p. 5) and this observation resonates with the significance of this first-step in the implementation plan. Also, with Figure 1, we have shared an example of how art-making may look like at this stage of the implementation. Each work of art created by the participants is their own way of conveying their perspective giving the activity an interesting and 'playful' twist (a twist that can be utilized for dialogic engagement). For example: Bhattacharya (in her paper - 2016) used a variety of objects in her artwork that included straw, wires, mirrors, peace symbols etc. The choices then served as a means to initiate and engage in dialogic conversation with her co-author. In regards to our framework, since the participants will thereafter use the art to write a narrative (ref: Label 2), the artwork's meaning (and composition) can/will continue to grow and evolve (as indicated by the 'multiple event' label in Figure 1).

\subsection{Personal Narratives using the Art they created: Label 2}

In their article, authors Stetsenko \& Ho note that "(f)or young children, it is a challenge to have their own voices heard, yet it is even a bigger challenge to have their voices forged, while establishing one's own stances from a relational yet authorial position" (p. 231-32). This observation serves as the primary motivation for this step in the framework implementation. For this step (ref: label 2), the students will first individually write 'narratives' around the topic/issue that the class is focusing on taking inspiration from their artwork they created. They will then share their narratives and the artwork with the other participants and engage in dialogic meaning-

\footnotetext{
${ }^{3}$ The issue can be from any subject area. For example, students in the science class can be exploring the issue of 'deforestation' and this issue can easily be adapted into the framework. Similarly, in a physics class students can be exploring about 'terraforming' and this issue too can easily be adapted into the framework. Likewise, students in a writing class who are to write about the issue of 'lexicon', too can easily use this framework.
} 
making (as shown in the example image in Figure 1). This will open the opportunity for the students to explain their perspective and for others to see and imagine perspectives across differences. In this stage the learners transform as both authors and audience and start to develop and recognize the existence of diversity in others' lived experiences. This step also allows for the sharing of "students' voices" and "interior lives" (Medina et al, 2018, p. 340-41) and this sharing is vital to the goal of developing diversity consciousness. In her paper, Bhattacharya (2016) shares her experience of using her art to write a narrative stating that "in writing these fragmented narratives, the narratives simultaneously write me" (p. 1114) and this observation articulately sums up the significance of this step in the framework.

\subsection{Drama, Writing-in-Role and Teotro: Label 3(a) and Label 3(b)}

This stage of our implementation framework is influenced ${ }^{4}$ by the work of authors Medina and Campano (2006). Both, the use of Drama and the use of Teatro (see labels 3(a) and 3(b)) present the opportunity to understand and interpret one's own experiences and that of others - opportunities that are essential in promoting empathy and awareness. Also, 'co-creating' and 'co-authoring' are shared themes between these imaginative practices and so they go hand-in-hand with the gist of our framework that is interested in the "consciousness as emerging within and out of shared activities with others" to further explore and forefront "social interconnectedness" (Stetsenko \& Ho, 2015, p. 226). As the labels indicate, both 3(a) and 3(b) can be approached in parallel within the framework before moving to the next step (label 4). For educators interested in 3(a), a simple approach to implementation can stem from borrowing and extending the 'Writing-IN-Role' model (ref: Table 1) implemented by Medina (2006 - where she uses fiction), one can instead utilize personal stories (of the students themselves ${ }^{5}$ produced in step 2 (label 2)) around common everyday activities/topics/issues as the starting point for the diversity consciousness activity. Herein, the 'hot seat' (ref: Table 1) activity can now allow for critical inquiry, reasoning and literacy into everyday activities/events through the lens of an 'other' helping the students to see/experience alternate realities and human experiences. Furthermore, as the students subsequently look beyond the story to speculate about the future, their work (imagination) can provide evidence for studying/observing/documenting the development (longitudinally) of diversity consciousness (especially about how they make connections of the new knowledge with their worldview). Similarly, for educators interested in 3(b), modifying and extending the 'Teatro' model (fictionalizing reality) implemented by Campano (2006), one can have several students play the same character simultaneously ${ }^{6}$ thus making each frozen moment (ref: Table 1) a 'multidimensional moment' where the same scenario can be analyzed simultaneously using different experiences/responses/voices. This too has the potential to allow for the development of critical inquiry, reasoning and literacy through the lens of an 'other' helping the students to see/experience alternate realities and human experiences. Most importantly, the 'multidimensional moment' approach can also help identify/document the 'similarities' between experiences while simultaneously educating the participants about their uniqueness as well.

In regards to the context of the imaginative practices suggested for Step 3 (labels 3(a) and 3(b)), Bhabha in his book 'The Location of Culture', discusses about the importance of "posing questions" about "solidarity and community from the interstitial perspective" (1994, p. 3). His discussion about Renee Green's approach of abandoning the 'identities of difference' to instead using creative intervention within in-between moments resonates with our project's theme of solidarity through understanding the differences via the similarities in the shared human experiences. The quote (in Bhabha's book) "It requires a person to step outside of him/herself to

\footnotetext{
${ }^{4}$ We would strongly encourage interested readers to first read the article by authors Medina and Campano before reading this section. It will help in better understanding this paper here forward.

${ }^{5}$ For examples look at https:// myimmigrationstory.com or https://childmigrantstories.com

${ }^{6}$ Observe that this is different from the "multiple viewpoints" model suggested as an extension on page 341 of the 'Performing Identities...' (2006) article. So, we believe that the 'multi-dimensional' moment model that we suggest is unique in regards to the extension of 'Teatro' for encouraging diversity consciousness.
} 
actually see what he/she is doing" (1994, p. 3) adds credence to the plan of using the multidimensional moment approach to Teatro for diversity consciousness. This also adds credence to the stance that “(i)n play and dramatic experiences, actors are also audiences to one another's performances. Co-players actively interpret an actor's actions; these interpretations in turn affect the way that actors enact scenes or authors craft scripts" (Medina, \& Wohlwend, 2014, p. 28).

\subsection{Playful Literacies, Story-telling and Re-Telling: Label 4}

Once the participants have completed steps/lables 1 through 3, they can now engage in step/label 4 which is primarily driven by dialogic engagements that forefronts the belief "that students' ideas will transform classroom discourse, other students' understandings, and even their own understandings" (Aukerman, 2013, p. 6). So, naturally the emphasis of this stage in the framework is to use playful literacies to forefront social interconnectedness. Here, storytelling and retelling can also be used to further encourage co-creating and co-authoring. In their article about playful literacies, authors Stetsenko \& Ho discuss the significance of allowing children to be "agentive actors in co-authoring themselves and their world" (2015, p. 221). The "transformative activist stance' proposed and defended in the article suggests reimagining playful literacies as a "pathway for children to discover how to co-author themselves and the world in a bidirectional spiral of mutual becoming" (2015, p. 225). The crux of the argument is that classroom activities should "offers unique opportunities for children to develop and exercise their agency, identity, and voice" (p. 221). The step/label 4 presented in the framework upholds this belief.

Another important aspect of this stage in the implementation of the framework is the act of story-telling and re-telling. In his paper, author Sumara discusses the significance of storytelling and re-telling/reading to meaning making. He states that "while it is interesting for readers to look back and notice how they have responded to characters and situations after their first pass through the text, it is more interesting to notice these markings during successive readings." (1998, p.206). He adds that "as readers come to identify with characters who are not themselves, and become involved in plots that are not their own, they must form alliances between their own experiences and those of the characters" (1998, p.206). This observation is vital to our framework. Since our framework encourages personal stories, the storytelling and re-telling (ref: label 4) will help the students 'connect' with their classmates more consciously and empathetically thus helping them redevelop their social imagination around diversity. Another important note from Sumara's paper that is worth noting in the context of our framework is that:

"It is during the practice of rereading that students begin to notice the traces of a "previous reader identity" in their text. As they move through the rereading experience, students become aware that, not only has the knowledge that emerged from their first reading shifted but, importantly, so too has their reading self" (1998, p.207).

\section{Discussion}

Producing and reading text is an integral part of the framework. As (can be) seen in the implementation flowchart (shown in Figure 1), writing personal narratives (ref: label 2) is an essential part of the process. Also, reading the narrative (ref: label $3 \mathrm{a}$ and $3 \mathrm{~b}$ ) to engage in dramatic modes are essential components of meaning-making towards the target goal of diversity consciousness. Taken together, there are several transitional zones within the framework that we will highlight/discuss in the sub sections below.

\subsection{Transitional Zone between Label 1 and Label 3}

The art that is produced by the students as part of the self-exploration (ref: Label 1) can be repurposed as "pre-text" to the other learners during the Writing-In-Role and Teatro phase of the project. This extends the usefulness of the art and also foregrounds meaning making in an integrated and conscious way into the flow of activities in the project implementation. For example, the pre-text can help the student in the 'hot-seat' to "re-imagine and re-contextualize 
what it is they are reading from the" art and the corresponding narrative "in relation to their own set of beliefs, attitudes and experiences" that "shifts the meaning of the text to become more personalized, social and relational". This 'shift' is the core of our reimagined classroom practices' objective since we are interested in moving away from "linear forms of interpretations" to instead encourage the emergence of meaning making in an "actively negotiated" encounter (Medina et al., 2018, p. 11).

\subsection{Transitional Zone between Label 2 and Label 3}

Between the 'writing personal narratives' (ref: Label 2) and the use of key drama practices (ref: Label 3), the personal narratives "can be retold over and over" among the group/learners and this can be utilized in the framework implementation to foreground the understanding of how "the context and conditions of reading alter the shape of the event of storytelling and the trajectory of meaning" (Sumara, 1998, p. 204). This allows for reimagining the classroom space where this rereading can take place to utilize reading as an "act of identity making" (Sumara, 1998, p. 205). This identity making can be further explored/studied during the writing-in-role,' the 'hot seat,' and the 'multi-dimensional moment' activities thus providing the teacher with a form of assessment tool as they make note of incremental progress within the implementation plan. This also aligns the primary view/intention (towards instilling diversity consciousness) with Dennis Sumara's view of identity making where identity is understood as "something that co-emerges with one's evershifting geographical, interpersonal, and intertextual experiences, and that identity is always the product of the interpretive work done around the continual fusing of past, present, and projected senses of self". For example, the 'multi-dimensional moment' will allow the students/learners "to look back and notice how they have responded to characters and situations after their first pass through the" text (enactment), subsequently it will be even "more interesting to notice these markings during successive" enactments since it will help study identity making/development with respect to diversity consciousness as a response to the engagements and activities (Sumara, 1998, p. 206).

\subsection{Transitional Zone between Label 3 and Label 4}

The concept of "horizontal reading" discussed by Dennis Sumara (1998) also fits in very well with how meaning making is understood and foregrounded in this framework. As already discussed (earlier in this section), the implementation encourages the use of immigrant stories as "horizontal reading." This horizontal reading can also allow for "juxtaposing" and "chaining" within the implementation plan to help develop "interpretive links" and "interpreted bridges" in the learners/students as they incrementally "respond to the text differently (with every pass) and, as a consequence, generate new knowledge and alter their reading identities" (Sumara, 1998, p. 207).

\section{Conclusion}

As can be seen from the implementation framework presented (in Figure 1) and discussed (in sections 3 and 4), 'dialogic comprehension-as-sensemaking pedagogy' is at the heart of the design. All throughout the implementation, the highlight is on the importance of the 'conversation' over the 'conclusion' indicating that the interest is in sensemaking pedagogy not simply as a matter of "nurturing and celebrating student understandings, but rather of engaging students in dialogue about text in which understandings are transformed through encountering the understandings of others" (Aukerman, 2013, p. 6). For example, the multi-dimensional moment approach "fosters refraction and interanimation of multiple readers' ideas" (Aukerman, 2013, p. 7). Also, the final phase of our project (ref: Label 4) emphasizes story-telling and retelling. This is evidence that the interest in sensemaking pedagogy is an essential part of cultivating diversity consciousness and the redevelopment of social imagination (that is inclusive of identity-making as well). The implementation is also "generative" and allows the "openness" necessary for "the active exploration of possibilities for meaning" (Aukerman, 2013, p. 5). By design, the teacher is positioned (as a co-participant) to support "radically different understandings" while also 
honoring "this kind of intellectual" meaning making even when it may (or may-not) align with his/her own thinking of sense-making (Aukerman, 2013, p. 5).

In summary, as already discussed in this document, the implementation plan/framework presented utilizes "reading comprehension as agentive meditational meaning making" to help create "new spaces as forms of participation in classrooms, centering students voices, where new forms of knowledge are made available and where complex remixings of texts and discourses emerge" (Medina et al., 2018, p. 2, p.18). It should be clear by now that the implementation plan affords the participants "the agency of discovery learning" and "the sanctity of free expression" (Medina \& Wohlwend, 2014, p. 26). Also, from the discussion presented earlier in this section it should be evident that the project is specifically designed to help learners discover how to be agentive actors. In conclusion, we will like to quote from authors Medina and Wohlwend's book (2014) regarding using drama and playful literacies that

"(o)nce a space was opened in the classroom to deliberately bring, improvise, and play around popular culture imaginaries, the students enacted a sense of agency and empowerment in collectively scripting, creating, and improvising other kinds of texts and identities" ( $p .90)$

The framework presented in this paper too aims at opening such a space in the quest to cultivate diversity consciousness alongside the production of new knowledge. We conclude this paper with the sincere hope that teacher-scholars and educators will find our ideas interesting enough to consider adapting and improving upon the framework that was presented in the paper.

\section{References}

Aukerman, M. (2013). Reading comprehension pedagogies: Toward a dialogical teaching ethic that honors student sensemaking. Dialogic Pedagogy: An International Online Journal, 1, 1-31.

Bhabha, H. K. (1994). The location of culture. London; Routledge.

Bhattacharya, K. \& Payne, R. (2016) Mixing mediums, mixing selves: arts-based contemplative approaches to border crossings, International Journal of Qualitative Studies in Education, 29(9), 1100-1117.

Bucher, R. D. (2000). Diversity consciousness opening our minds to people, cultures, and opportunities. Upper Saddle River, NJ: Prentice Hall.

Diamond, E. (1996). Performance and Cultural Politics, London and New York: Routledge.

Eranpalo, T., \& Jorgenson, J. (2018). Creating Dialogue in the Classroom. International Journal of Humanities and Social Sciences, 10(1), 1-13.

Greene, M. (1995). Releasing the imagination: essays on education, the arts, and social change. San Francisco: Jossey-Bass Publishers.

Hawkins, M. R. (2014). Ontologies of place, creative meaning making and critical cosmopolitan education. Curriculum Inquiry, 44(1), 90-112.

Hays, C. B. (2005, November). The Silence of the Wives: Bakhtin's Monologism and Ezra 7-10; Bakhtin and the Biblical Imagination Consultation; Philadelphia, PA.

Medina, C.L., \& Campano, G. (2006). Performing identities through drama and teatro practices in multilingual classrooms. Language Arts, 83(4), 332-341.

Medina, C., Perry, M., Lee, B., \& Deliman, A. (2018). Drama in Reading as meaning making practices: Trajectories and possibilities. (Draft Chapter).

Medina, C. \& Wohlwend, K. (2014). Literacy, play and globalization: Converging imaginaries in children's critical and cultural performances. New York: Routledge Research in Education Series.

Smagorinsky, P. (2001). If meaning is constructed, what is it made from? Toward a cultural theory of reading. Review of Educational Research, 71, 133-169.

Stetsenko, A., \& Ho, P. C. G. (2015). The serious joy and the joyful work of play: Children becoming agentive actors in co-authoring themselves and their world through play. International Journal of Early Childhood, $47(2), 221-234$.

Sumara, D. (1998). Fictionalizing Acts: Reading and the making of identity. Theory Into Practice, 37, 203-210. 
Van der Veen, C., Marjolein, D. \& Bert, O. (2018). Engaging children in dialogic classroom talk: Does it contribute to a dialogical self? In F. Meijers, F., \& Hermans, H (Eds.). The Dialogical Self Theory in Education, (pp. 49-63). Berlin: Springer International Publishing.

Zarnowski, M. (2009). The thought experiment: An imaginative way into civic literacy. Social Studies, 100(2), 55-62. 